\title{
DIAGNÓSTICO COPROPARASITOLÓGICO DE Fasciola hepatica EN GANADO BOVINO EN UNA EMPRESA PECUARIA CUBANA
}

\author{
Coproparasitological Diagnosis of Fasciola hepatica in Cattle in a Cuban \\ LiVESTOCK F ARM
}

\author{
Yuraimis Godoy M. ${ }^{1,2}$, Eungenio Roque L. ${ }^{3}$, Ingrid Doménech C. ${ }^{1}$, Rafmarys \\ Rodríguez F. ${ }^{4}$
}

\section{Resumen}

Con el objetivo de comparar el método coproparasitológico de sedimentación de Benedek con el kit inmunoenzimático FasciDIG para el diagnóstico de Fasciola hepatica en ganado bovino de una empresa pecuaria cubana, se realizó un estudio descriptivotransversal entre febrero y marzo de 2008. Se colectó muestras de heces a 254 bovinos Jersey, Holstein y mestizos. Se encontró $16(6.3 \%)$ animales positivos mediante el FasciDIG y $10(3.9 \%)$ por la técnica de sedimentación de Benedek. Se concluye que el método inmunoenzimático (FasciDIG) demostró mayor detectabilidad diagnóstica que el método de sedimentación.

Palabras clave: Fasciola hepatica, coprológico, inmunoenzimático, bovino

\section{Abstract}

The aim of the study was to compare the coproparasitological method of Benedek sedimentation against the immunoenzimeassay for the diagnosis of Fasciola hepatica in cattle from a Cuban livestock large farm. A descriptive cross-sectional study was conducted in February-March 2008 and faecal samples were collected to 254 Jersey, Holstein, and crossbreed animals. Sixteen positive cases $(6.3 \%)$ were detected using FasciDIG whereas only $10(3.9 \%)$ using the Benedek sedimentation method. It was concluded that the immunoassay method (FasciDIG) showed better diagnostic sensitivity than the Benedek sedimentation test.

Key words: Fasciola hepatica, conic cup, immunoenzymatic, bovine

\footnotetext{
${ }^{1}$ Instituto Medicina Tropical "Pedro Kourí”, La Habana, Cuba

${ }^{2}$ E-mail: godoy@ipk.sld.cu

${ }^{3}$ Facultad de Medicina Veterinaria y Zootecnia, Universidad Agraria de la Habana, Cuba

${ }^{4}$ Laboratorio Nacional de Parasitología Veterinaria, La Habana, Cuba
} 


\section{INTRODUCCIÓN}

La fascioliasis es una enfermedad parasitaria de distribución cosmopolita causada por tremátodos del género Fasciola. La especie más común, Fasciola hepatica, es un parásito digenético y hermafrodita que utiliza moluscos del género Limnaea como hospedero intermediario (Pérez-García et al., 2002). La forma adulta se localiza en los conductos biliares de mamíferos herbívoros y del hombre (Rodríguez et al., 2001; González et $a l .$, 2007), por lo que se le reconoce como una zoonosis emergente. La OMS ha estimado que existen, a nivel mundial, 2.4 millones de personas infectadas con Fasciola hepatica y unos 180 millones adicionales en riesgo de infección (Becerra, 2001).

Esta parasitosis representa un grave problema de salud animal, reportándose que existen 300 millones de bovinos y 250 millones de ovinos en riesgo de ser afectados, además de las pérdidas económicas que superan a los tres millones de dólares anuales a nivel mundial (López-Lemes et al., 2005). En Cuba, la fascioliasis es enzoótica del ganado bovino, constituyendo uno de los problemas más emergentes a resolver en la ganadería nacional. Se ha demostrado que el $35 \%$ de las pérdidas en el ganado adulto está ocasionado por la $F$. hepatica, y donde la pérdida por decomiso de hígados, tanto en la losa sanitaria como en el matadero, llega a ser del 70\%. El Instituto de Medicina Veterinaria, como institución rectora del trabajo veterinario en Cuba (IMV, 2007), reportó una infestación alta en bovinos, con una existencia de 1170 focos, 66969 enfermos y 3059 muertos.

El diagnóstico de fascioliasis en el animal vivo se basa en el hallazgo de huevos del parásito en heces; sin embargo, este método carece de sensibilidad en la fase aguda de la infección, debido a que el parásito se encuentra migrando por el parénquima hepático sin llegar a la madurez sexual, por lo que los exá- menes parasitológicos son negativos a la presencia de huevos. Por otro lado, durante la fase patente, la intermitencia en la expulsión de los huevos hace que también se presenten dificultades en el diagnóstico coprológico, por lo que se requiere de exámenes seriados Carrada, 2003; Daménech et al., 2009).

Debido a las dificultades que se presentan en el diagnóstico de esta trematodosis, se tiene como alternativa un método inmunoenzimático de detección de antígeno de excreción - secreción (Ag E/S) de $F$. hepatica (FasciDIG). Este Sandwich ELISA ha sido desarrollado por el Instituto Cubano de Medicina Tropical "Pedro Kourî" y tiene la facultad de detectar la infección activa en cualquiera de sus fases (Espino, 1997), con una sensibilidad del $95 \%$ y una especificidad del $99 \%$.

El presente estudio se llevó a cabo con el fin de comparar el método copropara-sitológico de sedimentación con el método inmunoenzimático FasciDIG para el diagnóstico de Fasciola hepatica en ganado bovino.

\section{Materiales y Métodos}

El estudio se realizó en la Empresa Pecuaria Genética «Niña Bonita» de la Provincia La Habana, Cuba, entre febrero y mayo de 2008. Se muestrearon 254 bovinos Jersey, Holstein y mestizos. El tamaño de la muestra se determinó utilizando como base el criterio establecido en el Programa de Lucha y Control de la Fascioliasis elaborado por el Instituto de Medicina Veterinaria de Cuba.

La toma de muestras se realizó entre las 05:00 y 07:00 horas, colectándose entre 10 y $20 \mathrm{~g}$ de heces del recto. Cada muestra se distribuyó en dos bolsas nylon para su análisis en los laboratorios del Centro Nacional de Parasitología (CNP) del Instituto de Medicina Veterinaria y del Instituto de Medicina Tropical "Pedro Kourî" (IPK), ambos en La Habana (IMV, 2007). 
Las muestras remitidas al CNP se analizaron mediante el método de sedimentación de Benedek (Ueno y Goncalves, 1998). El hallazgo de huevos en heces fue considerado como criterio positivo para el diagnóstico $F$. hepatica. En el IPK se procesaron las muestras empleando el kit diagnóstico "FasciDIG", el cual se basa en la detección de antígenos en heces (coproantígenos) usando el anticuerpo monoclonal ES-78 de subclase IgG2a, siguiendo la técnica descrita por Espino et al. (1992) y Espino y Finlay (1994).

Para que el inmunoensayo sea considerado válido, en el pozo correspondiente al control positivo debe aparecer una coloración naranja intensa cuyo valor de densidad óptica (DO) a $492 \mathrm{~nm}$ debe ser mayor o igual a 1.0, en tanto que el pozo correspondiente al control negativo debe permanecer incoloro, con DO menores a 0.24 . Se consideran positivas todas las muestras de heces cuyo valor DO sea mayor o igual a 0.24 (Espino et al., 1992, 2000).

\section{Resultados y Discusión}

Diez de las 254 muestras (3.9\%) analizadas mediante la prueba de sedimentación resultaron positivas a $F$. hepatica y 16 animales $(6.3 \%)$ resultaron positivos mediante el método inmunoenzimático FasciDIG.

La discrepancia en los resultados entre las dos técnicas podría deberse a que el diagnóstico de $F$. hepatica mediante la técnica de sedimentación (Copa Cónica), conocida como Regla de Oro, y utilizada de forma rutinaria en el diagnóstico por su bajo costo de ejecución, puede dar resultados negativos cuando la enfermedad se encuentra en la fase aguda, donde el parásito se halla migrando por el parénquima hepático sin llegar a la madurez sexual (Espino et al., 1997; Fredes et al., 1997; Sánchez et al., 2000). Además, existe una intermitencia en la excreción de los huevos de la $F$. hepatica adulta, ya establecida en el hígado, los cuales también pueden ser destruidos por las bacterias de la microbiota intestinal (Gorman et al., 1998). No obstante, Li et al. (2005) refieren que el muestreo coprológico seriado por animal puede aumentar la probabilidad de encontrar más animales positivos; sin embargo, los exámenes seriados consumen gran cantidad de tiempo, lo cual resulta desventajoso. Carrada (2003, 2007), por su parte, indica que para aumentar la capacidad de detección de la técnica con el rigor que exige la misma, es necesario tener en cuenta los factores que pueden influir en ella, tales como el horario de toma de muestra y el tiempo de espera entre decantación.

Es de importancia resaltar que la fase aguda de la enfermedad coincide con el periodo prepatente (periodo de tiempo que transcurre entre la ingestión de metacercarias y el inicio de la oviposición), el cual varía de acuerdo al hospedero y al número de parásitos que invaden los conductos biliares. Se ha demostrado que mientras mayor sea el número de parásitos que invaden el hígado, mayor será el tiempo que requieran en alcanzar los conductos biliares y la madurez sexual; por lo que, entonces, se alargaría el periodo prepatente $\mathrm{y}$, por tanto, la aparición de los huevos en las heces (Espino, 1997; Sandoval et al., 2003).

La explicación a un mayor nivel de detección de animales positivos por el método de inmunoensayo, se basa en que la detección de coproantígenos se hace posible desde que el parásito inmaduro se encuentra próximo a su localización definitiva en el conducto biliar y sus productos metabólicos son vertidos directamente al tracto gastrointestinal de forma constante, por lo que permite detectar la enfermedad en cualquiera de sus fases (Espino, 1997). La existencia de animales negativos por el método inmunoenzimático y positivos por el coproparasitológico, puede deberse a la carga parasitaria en hígado y a la concentración de antígenos excretados en heces, dando como negativos aquellos animales que se encuentran por debajo de su límite de detección (hasta 15 nanogramos) (Domenech et al., 2009). 


\section{Conclusión}

La aplicación del método inmunoenzimatico FasciDIG demostró mayor detectabilidad diagnóstica que la coprología.

\section{Literatura Citada}

1. Becerra RWM. 2001. Consideraciones sobre estrategias sostenibles para el control de Fasciola hepatica en Latinoamérica. Rev Col Cienc Pec 14(1): 28-35.

2. Carrada BT. 2003. Fasciolosis: diagnóstico, epidemiología y tratamientos. Rev Mex Gastroenterología 68(2): 3542.

3. Carrada BT. 2007. Fasciola hepatica: Ciclo biológico y potencial biótico. Rev Mex Patol Clin 54(1): 21-27.

4. Doménech CI, Marcet SR, Figueredo PM, Sarracent PJ. 2009. Conservación de heces humanas para la detección de antígenos de excreción-secreción de Fasciola hepatica. Rev Cubana Med Trop 61(2): 1-5

5. Espino AM, Finlay CM. 1994. Sandwich enzyme-linked immunosorbent assay for detection of excretory secretory antigens in humans with fascioliasis. J Clin Microbiol 32: 190-193.

6. Espino AM, Marcel R, Finlay CM. 1997. Fasciola hepatica: detection of antigenemia and coproantigens in experimentally infected rats. Exp Parasitol 85:117-120.

7. Espino AM. 1997. Inmunodiagnóstico de la fasciolosis humana y su aplicación en brotes epidémicos. Tesis de grado de Doctor en Ciencias Médicas. La Habana: Instituto Cubano de Medicina Tropical "Pedro Kourî". 82 p.

8. Espino MA, Borges A, Dumenigo EB. 2000. Coproantígenos de Fasciola hepatica de posible utilidad en el diagnóstico de la Fascioliasis. Rev Panam Salud Publica 7: 225-231.
9. Espino AM, Millan J, Finlay C. 1992. Detection of antibodies and circulating excretory-secretory antigens for assessing cure in patients with fascioliasis. Trans Roy Soc Trop Med Hyg 86: 649.

10. Fredes F, Gorman T, Silva M, Alcaino H. 1997. Diagnostic evaluation of chromatographic fractions of Fasciola hepatica by Western Blot and ELISA in infected animal. Arch Med Vet 29(2): 283-294.

11. González R, Pérez RM, Brito S. 2007. Fasciolosis bovina. Evaluación de las principales pérdidas provocadas en una empresa ganadera. Rev Salud Anim 29(3): 167-175.

12. Gorman T, Sánchez R, Fredes F, Alcaíno H. 1998. Inmunodiagnóstico de fasciolosis bovina mediante ELISA y Western Blot. Parasitol Día 22: 16-22.

13. [IMV] Instituto de Medicina Veterinaria de Cuba. 2007. Reporte anual de fasciolosis bovina en Cuba. Ciudad de La Habana; IMV.

14. Li EO, Leguía PG, Espino MA, Duménigo RB, Díaz EA, Otero $O$. 2005. Detención de anticuerpos y antígenos para el diagnóstico de Fasciola hepatica en alpacas naturalmente infectadas. Rev Inv Vet, Perú 16: 143-153.

15. López-Lemes M, Hernández S, Acuna AM, Nari A. 2005. Fascioliasis en la República Oriental del Uruguay. [Internet], [21 diciembre 2010]. Disponible en: http://produccionanimal.com.ar/ sanidad_intoxicaciones_metabolicos/parasitarias/parasitarias_bovinos/51fascioliasis_en_uruguay.htm

16. Pérez-García J, Álvarez-Sánchez MA, Mainar-Jaime RC, Rojo-Vázquez FA. 2002. Enfermedades parasitarias del ganado vacuno: métodos de control. Mundo Ganadero No 145. [Internet], [7 enero 2009]. Disponible en: http:// produccion-animal.com.ar/ sanidad_intoxicaciones_metabolicos/parasitarias/parasitarias_bovinos/39enfermedades_parasitarias_control.htm 
17. Rodríguez R, Torrado L, Pérez D, Morey F. 2001. Hematoma subscapular del hígado. Manifestación en tres pacientes con fasciolosis hepática. Rev Enf Inf Micro 21(3): 87-90.

18. Sánchez SS, Rojas OS, Reed SRG, Torres SMA. 2000. Fasciolosis hepatobiliar masiva. Rev Mex Gastroenterología 65(4): 83-179.
19. Sandoval E, Morales G, Jiménez D, Matinella L. 2003. Modificación de la prueba de precipitación circumoval para el diagnóstico inmunológico de Fasciola hepatica. Rev Cient Vet Trop 28(1): 25-36.

20. Ueno H, Goncalves PC. 1998. Manual para el diagnóstico de helmintosis de ruminates. $4^{\circ}$ ed. Salvador de Bahía, Brazil: 145 p. 